Revue d'histoire de l'Amérique française

RQ REVUE D.HISTOIRE DE L'AMÉRIQUE FRANÇAISE

\title{
Sources romaines pour l'histoire de l'Église catholique du Canada : le pontificat de Léon XIII (1878-1903)
}

\section{Monique Benoit et Matteo Sanfilippo}

Volume 44, numéro 1, été 1990

URI : https://id.erudit.org/iderudit/304864ar

DOI : https://doi.org/10.7202/304864ar

Aller au sommaire du numéro

Éditeur(s)

Institut d'histoire de l'Amérique française

ISSN

0035-2357 (imprimé)

1492-1383 (numérique)

Découvrir la revue

\section{Citer cette note}

Benoit, M. \& Sanfilippo, M. (1990). Sources romaines pour l'histoire de l'Église catholique du Canada : le pontificat de Léon XIII (1878-1903). Revue d'histoire de l'Amérique française, 44(1), 85-96. https://doi.org/10.7202/304864ar d'utilisation que vous pouvez consulter en ligne. 
NOTE DE RECHERCHE

\title{
SOURCES ROMAINES POUR L'HISTOIRE DE L'ÉGLISE CATHOLIQUE DU CANADA: LE PONTIFICAT DE LEON XIII (1878-1903) ${ }^{1}$
}

\author{
MONIQUE BENOIT \\ Archives nationales du Canada \\ MATTEO SANFILIPPO \\ Centre académique canadien en Italie
}

En 1898 Rome se décida, après bien des tergiversations, à créer une délégation apostolique permanente au Canada. Les rapports de Rome avec la hiérarchie de l'Eglise canadienne étaient devenus si enchevêtrés que la seule solution était d'aller voir sur place ce qui se passait réellement. Rapports, pétitions, plaidoyers et contre plaidoyers s'empilaient depuis des années sur le bureau du préfet de la Propagande. Ces documents décrivaient avec force détails les difficultés de l'Eglise canadienne dans ses rapports avec l'État, les inimitiés de plus en plus marquées entre Canadiens français et Canadiens anglais, et les tensions entre haut et bas clergé.

La volumineuse correspondance du premier délégué, Mgr Diomède Falconio, o.f.m., avec les évêques canadiens et avec Rome est rassemblée dans le fonds «Délégation apostolique du Canada» conservé aux Archives secrètes du Vatican. Ce fonds, enrichi de la correspondance des délégués successifs, porte sur les années 1899-1959².

1 Cet article est rédigé à partir de M. Benoit, Inventaire des principales séries de documents intéressant le Canada, sous le pontificat de Léon XIII (1878-1903), dans les archives de la Sacrée Congrégation «de Propaganda Fide» à Rome, Archives nationales du Canada, 1986, et de M. Sanfilippo, Inventaire des documents d' intérêt canadien dans l'Archivio Segreto Vaticano sous le pontificat de Léon XIII (1878-1903): Délégation apostolique du Canada, Délégation apostolique des Etats-Unis, Epistolae ad Principes et Epistolae latinae, et autres séries mineures, Archives nationales du Canada, 1987. Nous remercions le Conseil de recherches en sciences humaines du Canada, les Archives du Vatican et les Archives de la Propagande ainsi que le Centre académique canadien en Italie pour leur appui à ces projets.

2 La documentation relative aux délégués précédents envoyés pour régler des questions particulières, i.e. Mgr Merry del Val pour la question du Manitoba, se trouve répartie entre les archives vaticanes, série Epistolae ad Principes. Positiones et Minutae et l'Archivio per gli Affari Ecclesiastici straordinari. Une partie des renseignements contenus dans le présent article concernant la délégation apostolique a déjà été publiée sous une autre forme dans la publication du Centre de recherche en civilisation canadienne-française de l'Université d'Ottawa, Cultures du Canada français (automne 1988): voir M. Sanfilippo, «L'Archivio segreto vaticano et le fonds de la première délégation apostolique permanente au Canada (1899-1902)», 219-226. 
Par ailleurs, les Archives de la Propagande constituent une source plus considérable pour les années du pontificat de Léon XIII, tant par l'ampleur des séries que par la variété des sujets traités: entre autres, questions administratives, divisions et fondations de paroisses, redéfinition des frontières diocésaines. Aussi, examinerons-nous ces archives plus longuement dans le présent article. Les séries inventoriées traitent des efforts de réorganisation de l'Église à la suite de la croissance démographique et de l'ouverture de l'Ouest ainsi que des transformations sociales qui ont marqué ces années.

Les questions soumises à la Propagande sont souvent longues et délicates à résoudre tant les parties adverses sont résolues à obtenir gain de cause ${ }^{3}$. Par conséquent, les suppliques pour et contre les inévitables changements s'accumulent en de lourds dossiers tels la querelle de l'Université Laval ${ }^{4}$, la question des écoles du Manitoba ${ }^{5}$ et la division du diocèse de Trois-Rivières ${ }^{6}$, pour ne mentionner que les plus volumineux. Dans tous les dossiers, ce sont les évêques surtout qui écrivent pour demander directives et conseils, pour solliciter faveurs et privilèges pour le clergé et pour leurs diocésains. Mais les membres du clergé et des communautés religieuses, parfois les fidèles eux-mêmes, s'adressent aussi directement à la Propagande pour implorer justice contre leur évêque, pour s'opposer au démembrement de leur paroisse ou pour demander une faveur ou une dispense.

Les transformations de l'Église canadienne ne se font pas sans heurt, comme en témoignent les procès de nomination d'évêques et de création de diocèses. La situation est particulièrement difficile à TroisRivières lorsqu'il s'agit de diviser ce diocèse pour fonder celui de Nicolet contre le désir de Mgr Laflèche. L'évêque se soumet finalement à la volonté de Rome, mais non sans s'être longuement opposé à ce projet qu'il considère injuste et humiliant ${ }^{7}$. Par contre, en Ontario, Mgr Duhamel demande pendant plusieurs années la création du vicariat apostolique de Pontiac que réclament aussi les missionnaires de la Baie d'Hudson. Il l'obtient enfin en $1882^{8}$.

Ce qui saute d'abord aux yeux, c'est le grand nombre de demandes de dispenses matrimoniales pour affinité et consanguinité. Pour la seule

\footnotetext{
3 Voir M. Benoit, «Les tribulations des évêques canadiens», Annali Accademici Canadesi, 3 (1987-1988).

4 Archives «de Propaganda Fide», Rome (dorénavant APFR), Scritture originali riferite nei congressi generali (dorénavant $S O C G$ ), V.1020, ff 2-661; Scritture riferite nei congressi (dorénavant $S C$ ) V.27 à 29 (en entier).

APFR, Nuova Serie (dorénavant NS), V.240-241 (en entier), V.242, ff 1-469.

APFR, SOCG, V.1021, ff 519-938; SC, V.26 (en entier).

$7 S C$, V.26, nombreuses lettres de plaintes adressées à Léon XIII et PF. Voir aussi Nive Voisine, «La création du diocèse de Nicolet (1885)», Les Cahiers nicolétains, 5 (1983): 3-41 et 6 (1984): 147-214.

8 Voir APFR SOCG, V.1015(Ia) ff 186-383.
} 
année 1893, il n'y a pas moins de 475 demandes dont 30 seulement proviennent de diocèses extérieurs au Québec et, sur ces 30, 16 viennent du diocèse d'Ottawa ${ }^{9}$. Ces dispenses sont généralement accordées même si la réponse est parfois lente à venir. Dans certains cas, le refus de la Propagande est sans appel. Ainsi peut-on lire à l'endos d'une lettre de l'évêque de Trois-Rivières en 1881: on ne peut en aucun cas épouser sa belle-mère, ce genre de dispense ne s'accorde jamais, inutile d'insister ${ }^{10}$. Le secrétaire ponctue son commentaire d'un point d'exclamation, exprimant ainsi, fait exceptionnel, une opinion personnelle. Dans l'Ouest, les quelques causes de dispense portées à Rome sont presque toujours celles d'Amérindiens. En Ontario, sauf dans le diocèse d'Ottawa, les dispenses portent surtout sur des cas de diversité de culte.

Le Québec se distingue des autres provinces par la forte demande d'indulgences de toutes sortes, de bénédictions, de privilèges et de faveurs tant pour les prêtres et les religieuses que pour les paroissiens: qui veut un oratoire particulier, qui demande à conserver le saint sacrement dans sa chapelle, qui veut lire des livres à l'index ou encore ériger un chemin de la croix ${ }^{11}$. L'évêque expose ses doutes sur des questions liturgiques, sur le jeûne, sur les fêtes solennelles. Mgr Fabre, très consciencieux, demande en 1893 si une religieuse enfreint le jeûne en se lavant l'estomac avant la communion ${ }^{12}$. Prêtres et religieuses sollicitent des dispenses de voeux et, alors, la réponse peut tarder. Il faut souvent une seconde et même une troisième instance avant d'obtenir une réponse qui risque d'être un refus catégorique, surtout s'il s'agit de prêtres $^{13}$.

Les évêques prennent aussi souvent et longuement la plume pour plaider leur cause ou porter des accusations. Ces lettres peuvent aussi bien traiter de l'influence indue en politique que de la division des diocèses, de l'opposition des paroissiens à une décision de leur curé ou évêque, et des accusations de mauvais traitements lancées contre des prêtres ou des religieuses. Mais il ne faut pas croire que seuls les évêques du Québec se querellent, loin de là. Dans les provinces maritimes, les litiges se révèlent passionnants. Pendant qu'au Nouveau-Brunswick, les Acadiens réclament en vain des «évêques de notre race ${ }^{14}$, Mgr Hannan, évêque d'Halifax, appuie la guerre menée par le révérend D'Homme contre les Soeurs de la Charité d'Halifax. Il veut les voir fermer l'école et quitter la province. De longs rapports décrivent les

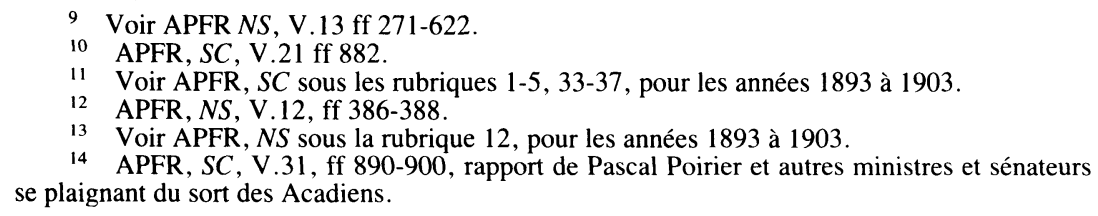


indignités qu'il fait subir aux religieuses au grand scandale des paroissiens. Mgr Hannan est appelé à Rome et sommé d'expliquer sa conduite et de produire un rapport annuel dont on lui dicte les termes précis. Sa mort en 1882 règle la question. Son remplaçant, natif du NouveauBrunswick alors que Mgr Hannan était Irlandais, saura mieux, espèret-on, ménager les susceptibilités de chacun ${ }^{15}$.

C'est donc dans un climat de mutations sociales, de grands projets et de querelles diverses qu'arrive Mgr Falconio, en octobre 1899, avec mission d'améliorer les relations entre le Saint-Siège et l'Église du Canada $^{16}$. Tout à fait inconnu au Canada et ne possédant qu'une connaissance très limitée de la situation politique et religieuse du pays, Mgr Falconio doit en outre affronter une forte opposition à sa mission considérée menaçante pour l'autonomie de l'Église et source de dépenses ${ }^{17}$. Mandaté à la fois par le Secrétariat d'Etat et par la Propagande, il doit, pour l'un, régler les controverses telle la question manitobaine $^{18}$, et, pour l'autre, améliorer les relations entre le Vatican et le gouvernement canadien. On lui demande en outre de servir de médiateur entre le haut et le bas clergé ainsi qu'entre les évêques d'expression française et anglaise, depuis longtemps à couteaux tirés ${ }^{19}$.

L'activité principale de Mgr Falconio, à en juger par l'abondance de documents, fut sans conteste la médiation entre les évêques canadiens et le clergé. Les simples soldats de la hiérarchie catholique avaient été les seuls à se réjouir de l'arrivée du délégué romain ${ }^{20}$. Le nombre de plaintes de curés contre leurs évêques contenues dans les archives de la délégation Falconio le confirme amplement ${ }^{21}$. De nombreux prêtres eurent recours à Falconio pour la moindre brouille avec leur ordinaire, ce qui embarrassa fort le délégué ${ }^{22}$. Il mit cependant peu de temps à discerner, dans l'amovibilité «sine causa» des curés, la racine du

15 Voir APFR, $S C$, V.21, ff 543-554, 767-770; V.22, ff 1-584.

16 Cf. APFR, NS, V.265, rubriques 154-1621903, ff 143-158; M. Sanfilippo, «La Santa Sede, il Canada e la Delegazione Apostolica ad Ottawa», Annali Accademici Canadesi, 2 (1986): 112-119. Pour une perspective plus vaste de la création de la délégation, cf. R. Perin, «La raison du plus fort est toujours la meilleure: la représentation du Saint-Siège au Canada, 1877-1917», La Société d'histoire de l'Église catholique. Sessions d'études, 50 (1983): 99-117.

17 Depuis la création de la Délégation apostolique aux États-Unis, les évêques du Canada s'inquiétaient du coût élevé de l'installation d'une délégation permanente. Cf. Archives secrètes du Vatican (dorénavant ASV), Délégation apostolique des États-Unis (dorénavant DAEU) VI, chemise 22, lettres de Mgr Satolli à Mgr Fabre). L'épiscopat canadien dut faire don d'une résidence au délégué, cf. ASV, Letter Book of Mgr Falconio (dorénavant LBF), nos 28, 45-46, 5354 , et payer les taxes, cf. APFR, NS, V.265, rubriques 154-162, ff 270-271. Pour une explication de la résistance "politique» à la création d'une délégation permanente, $\mathrm{cf}$. N. Voisine, «Rome et le Canada: la mission de Mgr Conroy», Revue d'histoire de l'Amérique française, 33,4 (mars 1980): 499-519.

18 Cf. Leonis XIII Pontificis Maxim, ACTA, 19 (Romae 1900): 128-130.

19 Cf., supra, note 17.

20 ASV, DAEU, section $X$, chemise 66 $88,157$.

1 Cf. ASV, Délégation apostolique du Canada (dorénavant DAC), 5, 7, 13, 26, 28-29,

22 Cf. ASV, DAC, 5, chemise «Rimouski: abbé Fraser vs évêque de Rimouski». 
problème et chercha à sensibiliser la Propagande à ce sujet, mais les évêques s'opposèrent fermement aux solutions proposées ${ }^{23}$.

Les documents révèlent aussi que les disputes entre les évêques et le bas clergé étaient souvent à l'origine de litiges entre évêques et paroissiens. Dans certains cas, les fidèles défendaient leur curé, dans d'autres, ils refusaient catégoriquement le démembrement de leur paroisse $^{24}$. Par ailleurs, les griefs de paroissiens au sujet de la moralité peu édifiante de leur curé n'étaient pas rares. Mais les plaintes les plus graves avaient trait à l'ingérence de l'Église dans les affaires politiques fédérales et provinciales ${ }^{25}$. Ces doléances étaient évidemment liées à l'affrontement des deux groupes linguistiques de la hiérarchie catholique et surtout à la question du Manitoba. Bien décidé à mettre fin à la propagande de l'épiscopat en faveur des Conservateurs ${ }^{26}$, le Parti libéral mit tout en oeuvre pour convaincre le Saint-Siège de nommer un délégué chargé de mettre bon ordre à la situation.

La délégation apostolique permanente avait été créée dans l'espoir d'éviter que ne se reproduise dans les autres provinces une situation semblable à celle qui avait déchiré le Manitoba. Comme Benoit XV devait le réaffirmer en 1916, Rome ne comprenait ni ne pouvait tolérer les divisions linguistiques ou ethniques au sein de l'Église ${ }^{27}$. Sur ce terrain, Mgr Falconio n'eut pas la partie facile. Où qu'il allât au pays, il était confronté à ces questions. Dans les provinces maritimes, la question acadienne n'occupait pas seule la scène ${ }^{28}$. De virulentes querelles divisaient le clergé d'origine irlandaise et écossaise ${ }^{29}$. Au Québec, les minorités anglophones cherchaient à s'approprier autant de privilèges que possible, cependant que les catholiques d'origine italienne tenaient mordicus à leurs propres traditions ${ }^{30}$. En Ontario, un conflit couvait depuis des décennies entre les catholiques francophones et anglophones tant au sein du clergé que parmi la population, comme l'illustrent les luttes pour le contrôle des diocèses et de l'Université d'Ottawa ${ }^{31}$. Dans

23 Cf. ASC, LBF, nos 950, 1140, 1224.

24 Cf. ASV, DAC, 13, chemise «St John: divisione parrochia», et 26, chemise «Sherbrooke: parrochia di Shifton, 1901-1902».

${ }_{25}$ Cf. ASV, DAC, 13, chemises «Antigonish: agitazione politica...» et «Antigonish: parrocchia di Heatherton»; DAC 69, LBF, nos 318 et 496.

${ }_{26}$ Cf. Perin, «La raison du plus fort...»; Louis Garon, «Inventaire analytique du fonds Félix-Gabriel Marchand», Rapport de l'Archiviste de la Province de Québec, 52 (1874): 146, $156,163$.

27 Cf. N. Voisine, éd., Histoire du catholicisme québécois, tome 3; J. Hamelin et $\mathrm{N}$. Gagnon, Le XXe siècle, tome 1: 1898-1940 (Montréal, Boréal Express, 1984), 98-99.

28 Cf. ASV, DAC, 178, chemise «La questione acadese during the time of Mons. Falconio", et LBF, nos 34 et 1058 .

29 Cf. ASV, DAC, 6, chemise "Charlottetown: sopra la condizione di sacerdoti irlandesi nella diocesi di Charlottetown».

30 Cf. R. Perin, «Religion, Ethnicity and Identity: Placing the Immigrant Within the Church», Canadian Issues/Thèmes canadiens, 7 (1985): 212-229.

31 Cf. R. Choquette, L'Église catholique dans l'Ontario français du dix-neuvième siècle (Ottawa, Éditions de l'Université d'Ottawa, 1984), 223-312; ASV, DAC 50, plusieurs chemises, et LBF, nos $1249,1264,1370,1427,1471,1472,1582$. 
l'Ouest, l'archevêque de Saint-Boniface, sourd aux directives de Rome et de son délégué, poursuivait sa propre politique qui allait à l'encontre de la stratégie libérale appuyée par Mgr Falconio et ses supérieurs ${ }^{32}$. La question scolaire, débordant les frontières du Manitoba, touchait aussi les territoires du Nord-Ouest, le Yukon et l'Athabaska. De plus, le refus des Ukrainiens de s'adapter au rite latin et la nécessité de pourvoir aux besoins matériels de ces immigrants posaient à l'Église un surcroît de problèmes ${ }^{33}$. Et enfin, en Colombie Britannique, les catholiques de langue anglaise du diocèse de New Westminster protestaient avec une telle véhémence contre le transfert de leur diocèse à l'archevêque de Saint-Boniface qu'il fallut se résoudre à créer, à l'extrémité du pays, une province ecclésiastique autonome ${ }^{34}$.

L'oeuvre de médiation de Mgr Falconio n'avait, malheureusement, aucune chance de succès. Il ne se faisait d'ailleurs pas d'illusion. Six mois à peine après son arrivée au Canada, il tentait d'expliquer à ses supérieurs l'impasse dans laquelle il se trouvait. D'une part, les évêques ne voulaient renoncer à leur pouvoir sur le bas clergé et, d'autre part, la question du Canada français promettait de s'éterniser. On peut sentir que Mgr Falconio était sensible à la lutte des Canadiens français pour défendre leur langue, mais des raisons d'ordre ecclésiastique lui déconseillaient de prendre leur part. La seule entorse à cette règle fut son intervention en faveur de l'Université d'Ottawa. Il craignait que les revendications linguistiques ne détruisent la cohésion de l'Église et n'affaiblissent l'esprit de soumission ${ }^{35}$.

\section{DESCRIPTION DES SÉRIES}

\section{A - Archives de la Propagande}

La documentation intéressant le Canada aux Archives de la Propagande se trouve dans les séries suivantes:

1. ACTA, 1878-1903, contenant les procès et les décisions prises sur les grandes questions.

2. SCRITTURE ORIGINALI RIFERITE NEI CONGRESSI (SOCG), 1878-1892, renferme la documentation originale dont une partie a servi à former les dossiers des procès.

3. SCRITTURE RIFERITE NEI CONGRESSI (SC), 1878-1892, porte sur les questions quotidiennes des missions.

4. LETTERE

34 APF, ACTA, V.274 (1903) ff 311-325; voir aussi ASV, DAC, 95, chemise «Progetto per l'erezione di une provincia ecclesiastica nella Colombia Inglese». Les évêques du Québec demandaient depuis longtemps la réunion de la Colombie Britannique à l'archevêché de Québec ou, plus tard, à celui de Saint-Boniface, cf. APFR, SOCG, V.1000, ff 280-281.

35 Ibid., no 1058 . 
5. NUOVA SERIE (NS), 1893-1903, la nouvelle série où sont fondues les séries SOCG, SC et LETTERE.

6. UDIENZE

\section{1 - La série ACTA}

Les quatre premières séries, qui sont aussi les plus importantes et les plus volumineuses, ont été inventoriées. Dans la série ACTA se trouvent réunies les minutes des assemblées générales (ponenze) de tous les cardinaux de la Sacrée Congrégation de Propaganda Fide, tenues une fois par mois et portant sur les nominations d'évêques et de coadjuteurs, la création et la division de diocèses, certains litiges, l'approbation des règles et constitutions de communautés religieuses, l'approbation des décrets des conciles provinciaux. Chaque dossier contient généralement trois éléments principaux: le résumé de la cause, rédigé en italien par le secrétaire, le rescrit, en latin, portant la décision prise par l'assemblée, et le sommaire des documents à l'appui, ou actes du procès. Cette partie peut être rédigée en français, en italien ou en latin. Beaucoup de dossiers contiennent aussi l'opinion des consulteurs, plus souvent qu'autrement en latin. Les documents de cette série sont imprimés. Jusqu'en 1892, chaque dossier ACTA correspond à un dossier de la série SOCG contenant la documentation manuscrite originale, et portant le même numéro dans l'inventaire. À compter de 1893, la documentation originale se trouve dans la série NS.

\section{2 - La série SOCG}

La série SOCG correspond à la série ACTA et la complète. Les documents originaux conservés dans cette série sont divisés en deux groupes. Le premier groupe comprend les documents retenus pour la ponenza et dont on retrouve copie, soit en traduction soit en version originale, dans le sommaire imprimé de ACTA. Chacun de ces documents porte un chiffre romain ou une lettre majuscule, à l'en-tête ou à la fin, correspondant à sa place dans le sommaire. Le second groupe est constitué des documents supplémentaires, ceux qui n'ont pas été utilisés pour les procès. On en retrouve une quantité considérable dans certains dossiers: voir, entre autres, la querelle de l'Université Laval et de l'École de médecine de Montréal, ou encore la question des biens des Jésuites ${ }^{36}$. À la fin de chaque document, un bref paragraphe en décrit le contenu, avec plus ou moins de détails selon le cas, ne donnant bien souvent que le sujet général. Les dossiers de cette série contiennent habituellement, outre la documentation mentionnée, une copie entière ou partielle de la ponenza. Les lettres originales en anglais sont toujours accompagnées d'une traduction italienne ou française. Dans l'ensem-

36 APFR, SOCG, V.1029(IIa) ff 1-863. 
ble, les dossiers sont complets mais, pour l'année 1880, tous les originaux manquent. Pour ceux-ci et quelques autres il ne reste donc que les ponenze dans ACTA.

\section{3 - La série SC}

Les questions, requêtes et problèmes présentés dans les documents de la série SC étaient discutés au cours des réunions hebdomadaires de la Sacrée Congrégation de Propaganda Fide. Ils portent sur le quotidien de la vie des missions: les demandes de dispenses de mariage et de voeux, les requêtes d'oratoires privés et de facultés, les rapports des anciens du Collegio Urbano ${ }^{37}$, les plaintes des prêtres contre leur évêque, les querelles, les lettres de félicitations ou de remerciements pour faveurs obtenues, les demandes de renseignements, de subventions, de directives, de livres religieux, d'objets sacrés et tant d'autres, en passant par les rapports des diocèses et des communautés religieuses. On y trouve aussi des dossiers volumineux comme la question de la division du diocèse de Trois-Rivières qui occupe un volume entier de 1405 folios $^{38}$ ou encore la question de l'Université Laval en trois volumes totalisant 3565 folios $^{39}$.

La série SC est divisée en deux parties: la première selon une division géographique et la seconde par matières. Presque tout ce qui intéresse la Canada se trouve dans la sous-série America Settentrionale Canada, Nuova Bretagna, Labrador, Terra Nuova. Pour les années du pontificat de Léon XIII, treize volumes portent entièrement sur le Canada, sauf quelques rares documents placés là par erreur et concernant les État-Unis ou d'autres pays. Par contre, plusieurs notes renvoient à des documents d'intérêt canadien placés dans d'autres sousséries. Étant donné la proximité des États-Unis, la confusion entre frontières civiles et frontières ecclésiastiques dans l'Ouest, et surtout l'appartenance de Vancouver à l'archidiocèse d'Oregon jusqu'en 1903, des documents d'intérêt canadien se trouvent aussi dans la sous-série America Centrale dal Canadà all' Istmo di Panama. C'est là qu'est conservée une grande partie de la documentation sur le diocèse de Vancouver. Quant aux sous-séries par matière, aucune n'a encore été inventoriée, mais de prime abord et selon la description qu'en fait le père Joseph Metzler ${ }^{40}$, elles semblent contenir peu de matériel relatif au Canada.

\footnotetext{
37 Voir à ce sujet Giovanni Pizzorusso, «Le «Lettere di Stato»: una fonte documentaria dell'Archivio della Congregazione 'de Propaganda Fide' di particolare interesse canadese (18931908)», Annali Accademici Canadesi, 5 (1989): 101-114. Cet article constitue la première étape d'un travail de recherche sur les rapports personnels entre certains prélats et prêtres canadiens et la Propagande.

38 APFR, $S C$. V.26

39 APFR, SC, V.27 à 29

$40 \mathrm{~J}$. Metzler, Inventory of the Historical Archives of the Sacred Congregation for the Evangelization of Peoples or «de Propaganda Fide» (Rome, Urbaniana University Press, 1983).
} 


\section{4 - La série LETTERE}

La série LETTERE est un registre de lettres de la Propagande en réponse aux demandes qui lui sont adressées. Cette série se termine en 1892. À partir de 1893 ces lettres, sous forme de brouillons rédigés en latin, sont incluses dans la série NS. À la disposition du chercheur, l'index de la série s'intitule Indice delle lettere, parte occidentale (pour les années 1878 à 1892 , voir les volumes 32 à 40) et contient un bref résumé de chaque lettre, le nom du destinataire, l'année et la page du document en question. Pour les décrets inclus dans cette collection il existe également un index en trois volumes commençant en 1719.

\section{5 - La série NS}

La NUOVA SERIE rassemble, à partir de 1893, les séries SOCG, SC et LETTERE. Pour les années 1893 à 1903, elle comprend 265 volumes, environ 26 pour chaque année. La documentation y est classifiée par rubriques réparties sous des numéros de code allant de 1 à 62 pour les matières et de 100 à 162 pour les divisions géographiques. Par exemple, aux numéros de code allant de 6 à 9 sont regroupées les rubriques se rapportant aux collèges, séminaires, universités; aux numéros 12 et 13 se trouvent les rubriques se rapportant aux questions matrimoniales, c'est-à-dire aux demandes de dispenses de mariage. On trouve des documents concernant le Canada sous toutes les rubriques matières. Sous les rubriques pays, par contre, seuls le Canada et les États-Unis nous intéressent. Au numéro 154, sous la rubrique America, Canadà e possedimenti britannici, sont répertoriés, entre autres, les gros dossiers qui correspondent aux ponenze de ACTA à compter de 1893: divisions de diocèses, nominations d'évêques et ainsi de suite. Vancouver cependant, parce que suffragant d'Oregon, vient sous la rubrique America, Stati Uniti portant le numéro de code 153. Chaque dossier est placé à la date du dernier document. Ainsi, par exemple, la question du Manitoba - deux volumes et demi totalisant 2400 folios - est placée à la date 1902 et contient tous les documents sur la question envoyés à la Propagande à partir de $1894^{41}$.

\section{6 - La série UDIENZE}

Enfin, il y a la série UDIENZE. Lors d'audiences avec le pape, le cardinal préfet de la Propagande présentait certaines requêtes d'ordre personnel: demandes de faveurs, d'indulgences, de privilèges, de dispenses et autres. Les décisions du pape se trouvent dans les minutes de la série ACTA. Cette série n'est donc pas essentielle et elle a peu de valeur du point de vue historique. Elle était importante cependant pour le fonctionnement de la Sacrée Congrégation. Pour ceux qui désirent la

\footnotetext{
41 APFR, NS, V.240 à 242.
} 
consulter, il existe un bon index général en 28 volumes couvrant toute la période de son existence, de 1666 à 1897, les six derniers volumes, 23 à 28, portant sur les années 1867 à 1897.

Les documents conservés aux archives de la Propagande sont reliés, divisés selon l'année et le sujet, et contiennent entre 500 et 1000 folios. La pagination des volumes d'archives est souvent double: une manuscrite, plus ancienne, et l'autre, plus récente, imprimée. De tous les volumes compilés, un seul est en liasse et non paginé.

L'italien et le français sont les langues les plus fréquemment utilisées à la Propagande. Aussi, les documents en anglais sont-ils accompagnés d'une traduction en général italienne, parfois française. Dans certains dossiers contenant une volumineuse correspondance en anglais, on trouve même les factures du traducteur. Mais, dans l'ensemble, les évêques qui écrivaient en anglais le faisaient par l'intermédiaire du Collège irlandais à Rome. Son directeur, Mgr Kirby, transmettait luimême la requête en une traduction libre italienne, conservant chez lui, plus souvent qu'autrement, l'original. Plusieurs évêques des provinces maritimes avaient étudié au Collegio Urbano: Mgrs O'Brien, Cameron, Howley, MacDonald, entre autres. Ceux-ci correspondent toujours en italien comme le font d'ailleurs tous les anciens étudiants de ce collège lorsqu'ils doivent faire rapport de leur état, mission et situation personnelle en vertu d'un serment fait à la Propagande.

En règle générale, les évêques d'expression française écrivent en français. Fait exception le cardinal Taschereau qui corrrespond presque exclusivement en latin. Les requêtes de dispenses de mariage sont habituellement en latin ainsi que les actes et décrets des conciles et certaines parties des ponenze. Les évêques du Québec semblent s'adresser directement à la Propagande plus volontiers et plus souvent que ne le font leurs collègues des autres provinces. Cependant, il arrive aussi que des requêtes, particulièrement dans les cas de demandes de facultés, soient faites par l'intermédiaire d'une communauté religieuse à Rome.

\section{$B$ - Archives secrètes du Vatican}

Le fonds de la Délégation apostolique du Canada dans l'Archivio Segreto Vaticano contient 772 boîtes de documents et les registres de correspondance de tous les délégués de 1899 à 1959. Dans le Letter Book of Mgr Falconio, 1899-1902, on retrouve le résumé, et quelquefois le texte intégral, de toutes les lettres du délégué ainsi que plusieurs biographies dont celles de Mgr Falconio lui-même, auxquelles furent ajoutées les notices nécrologiques et les biographies de ses trois secrétaires: Marie-Étienne Clavette, Edward Fisher et Francis Solanus Schaefer. 
Les boîtes de documents sont numérotées de 1 à 775 . Notons que les boîtes 565 à 567 manquent. Chaque boîte contient plusieurs chemises sur des sujets divers. Une seule exception, la boîte 69, qui renferme un bloc de documents et de coupures de journaux sur la question scolaire au Manitoba. Chaque chemise constitue un dossier relatif à un individu, une congrégation religieuse, une paroisse. Une brève inscription rédigée soit en italien ou en français, soit en anglais ou en latin ou bien encore en un mélange de deux ou trois langues, en révèle le contenu. Les documents ne sont pas numérotés mais disposés selon un ordre plus ou moins chronologique.

Les documents de la délégation Falconio sont conservés dans les boîtes 5 (diocèse de Chicoutimi), 13 (diocèses des provinces maritimes), 26 (diocèses de Montréal, Saint-Hyacinthe, Sherbrooke, Valleyfield), 37 (diocèse d'Ottawa), 50 (diocèses de Québec, Ottawa, Pembroke, Saint-Boniface, Saint-Albert, Saskatchewan, Yukon, Vancouver), 69 (question scolaire du Manitoba), 88 (diocèses de l'Ontario), 95 (diocèses de New Westminster et de Vancouver), 114 (question acadienne), 115 (premier Concile du Canada), 157 (religieux et religieuses), 178 (correspondance avec la Propagande et le Secrétariat d'État, relations sur la propagande des protestants au Canada et sur l'état de l'Église catholique canadienne, lettres aux évêques du Canada, questions concernant les Ruthènes et les Acadiens), 179 (relations et statistiques au sujet des écoles séparées).

Si la correspondance de Mgr Falconio et de ses secrétaires est consignée en bon ordre dans le Letter Book of Mgr Falconio, il n'en va pas de même du reste de la documentation. Se retrouvent en effet pêlemêle les lettres du clergé et des fidèles canadiens, nombre de télégrammes et de mémoires, des copies de lettres et de documents plus anciens, des attestations, des certificats, des statistiques, des biographies, des résumés de causes civiles et ecclésiastiques, des coupures de journaux, des livres et des opuscules, quelques photographies et des cartes géographiques.

\section{C - Autres séries}

Les séries inventoriées aux Archives de la Propagande et aux Archives secrètes du Vatican ne constituent pas l'entière documentation sur l'histoire des délégations apostoliques. Par exemple, certains aspects de la question du Manitoba en rapport avec la mission de Mgr Merry del Val ne peuvent être éclaircis qu'en consultant d'autres archives. On trouvera, entre autres, dans la série vaticane Epistolae ad Principes. Positiones et Minutae, de nombreux documents traitant du problème 
du Manitoba ${ }^{42}$. Par ailleurs, il est possible de suivre la mission de $\mathrm{Mgr}$ Merry del Val dans l'Archivio per gli Affari Ecclesiastici straordinari ${ }^{43}$.

Mentionnons, en outre, que le fonds Segreteria di Stato des Archives secrètes du Vatican contient des lettres de Mgrs Taschereau, Fabre, Laflèche, Bégin et Bruchési. Aux mêmes archives, il faudrait aussi consulter la correspondance de Mgr Laflèche avec le cardinal Oreglia di Santo Stefano conservée dans le fonds Spogli dei Cardinali ${ }^{44}$.

Les fonds que nous avons présentés constituent une source unique pour l'étude des relations entre Rome et le Canada. Ils donnent aux chercheurs la possibilité de brosser un tableau détaillé des problèmes de l'Église canadienne, d'étudier les conflits entre les groupes ethniques, tant au sein de l'Église que de la population, et d'éclaircir des débats encore mal connus. Bien que les archives vaticanes ne puissent être considérées comme la seule source valable pour l'histoire religieuse du Canada, il est certain qu'elles constituent un lieu d'observation privilégié. Elles offrent en outre le très grand avantage de rassembler en un seul endroit une quantité considérable de documents sur tous les diocèses du Canada ${ }^{45}$.

42 Cf. ASV, Epistolae ad Principes. Positiones et Minutae, documents relatifs à l'élaboration de l'encyclique Affari Vos: vol. 133, ff 222r-273r; vol. 138, chemise «8 dicembre 1897. Canada. Manitoba. Enciclica Affari Vos»; vol. 143, chemise «B: Missive Appunti Annotazioni senza data»; vol. 148, chemise «B: missive, note, appunti».

43 Particulièrement intéressant pour la question du Manitoba, la mission canadienne de Mgr Merry del Val et les problèmes relatifs à l'ingérence politique du clergé canadien. Pour l'inventaire de la période du pontificat de Léon XIII, voir aux Archives nationales du Canada: N. Serio et L. Bruti Liberati, Inventaire des "Canadiana» dans les Archives du Saint-Siège 1878-1903.

${ }_{44}$ Enfin signalons l'ouvrage récent de Roberto Perin Rome in Canada. The Vatican and Canadian Affairs on the Late Victorian Age (University of Toronto Press, 1990). Il s'agit d'une étude détaillée qui analyse les divers aspects des missions de Mgrs Conroy, Merry del Val et Falconio au Canada. L'auteur a puisé une large part de sa documentation dans les archives romaines.

45 Les Archives du Vatican et les Archives de la Propagande sont ouvertes aux chercheurs jusqu'à la fin du pontificat de Benoît XV, c'est-à-dire jusqu'en 1922. Actuellement, Matteo Sanfilippo et Giovanni Pizzorusso préparent un inventaire des documents d'intérêt canadien pour les années 1903-1914 (pontificat de Pie X) aux ASV. Aux Archives de la Propagande, Giovanni Pizzorusso a terminé un inventaire pour les années 1904-1914; il espère entreprendre bientôt l'inventaire de la période 1914-1922. 